\title{
Neutral Gas in Resonance-Ring Galaxies
}

\author{
Stuart Ryder, Ronald Buta, and Hector Toledo ${ }^{1}$ \\ Department of Physics 8 Astronomy, University of Alabama, \\ Tuscaloosa, AL 35487-0324
}

\begin{abstract}
We have mapped the distribution and kinematics of $\mathrm{H} \mathrm{I}$ in NGC 1433 and NGC 6300 with the Australia Telescope Compact Array, in order to test the association between resonances and ring phenomena. We find a bar pattern speed in NGC 1433 of $\Omega_{\mathbf{p}}=26 \pm 5 \mathrm{~km} \mathrm{~s} \mathrm{kpc}^{-1}$, and postulate that $\mathrm{NGC} 6300$ is still evolving to a mature resonance-ring galaxy.
\end{abstract}

\section{Introduction}

Although both theoretical models (Schwarz 1981) and observational statistics (Buta 1995) strongly suggest a link between stellar ring features and specific resonances with the bar angular pattern speed, a rigorous test of the resonancering theory really requires a complete knowledge of the galaxy's velocity field. H I studies of a handful of ringed barred spiral galaxies (e.g., NGC 1291 and NGC 5101 [van Driel et al. 1988]; NGC 4736 [Mulder \& van Driel 1993]; NGC 2273, NGC 4826, and NGC 6217 [van Driel \& Buta 1991]; NGC 1398 [Moore, these proceedings]; and NGC 5850 [Higdon \& Buta, these proceedings]) have generally shown concentrations of $\mathrm{H}$ I gas coincident with the optical rings/ pseudorings, with central holes as large as the bar (if there is one). However, none of these galaxies contains all three of the ring types observed (nuclear, inner, and outer (pseudo)rings), and patchy velocity field coverage requires that certain assumptions be made about the rotation curve.

Until the recent commissioning of the Australia Telescope Compact Array (ATCA), two of the very best examples of ringed barred spirals in the entire sky were inaccessible to existing aperture synthesis telescopes. NGC 1433 is the prototype of the ringed barred spirals, having as it does a nuclear ring, an inner ring, and an outer pseudoring, besides being one of the nearest and best presented. Buta (1986) presented UBVRI surface photometry and $\mathrm{H} \alpha$ kinematics for NGC 1433, but was unable to unequivocally associate the rings with particular resonances. In contrast to NGC 1433, NGC 6300 has only a single broad and dusty inner ring, but with a similar distance, angular size, and Hubble type to NGC 1433, it makes for an ideal comparison. Buta (1987) inferred noncircular motions in the bar of NGC 6300 from emission-line spectroscopy, and

\footnotetext{
${ }^{1}$ Also at Instituto de Astronomia-UNAM, México
} 
pointed to an apparent misalignment between its bar and inner ring major axes, which in most ringed barred spirals are parallel to each other.

The primary motivations for our study were to use both our knowledge of the resonant orbit locations and the disk kinematics to determine the bar pattern speed, a crucial parameter in dynamical models which is almost impossible to measure otherwise. We also wish to test the predictions of Schwarz (1981), Contopoulos \& Papayannopoulos (1980), and others that the actions of a strong bar will lead to gas deficiencies near the $L_{4}$ and $L_{5}$ points at corotation, as well as non-circular motions in the bar region itself.

\section{Observations}

We observed both galaxies for a total of approximately 60 hours each, using five different configurations of the ATCA, with maximum baselines up to $1.5 \mathrm{~km}$. Visibility data was recorded both within an $8 \mathrm{MHz}$ bandwidth of the redshifted $\lambda 21 \mathrm{~cm} \mathrm{HI}$ emission lines, as well as in a $128 \mathrm{MHz}$ bandwidth in the nearby continuum at $1.38 \mathrm{GHz}$. Each configuration was edited and calibrated separately using the AIPS software package, after which one master $u v$-dataset was constructed for each galaxy. Continuum images, or channel maps of an $\mathrm{H}$ I emission line cube, of $256 \times 25610^{\prime \prime}$ square pixels were constructed from a weighted Fourier transform of the $u v$-dataset, and then the images and channel maps were CLEANed down to a level of $0.15 \mathrm{mJy}^{\text {beam }}{ }^{-1}$ and $1.0 \mathrm{mJy}$ beam $^{-1}$ respectively. The results of the continuum observations are discussed separately by Shukla \& Ryder (these proceedings). Moment maps of H I column density, velocity, and velocity width were extracted from the datacubes. With a uniform weighting scheme, the channel maps have a synthesized beamwidth $\sim 30^{\prime \prime}$, and are separated by $10 \mathrm{~km} \mathrm{~s}^{-1}$.

\section{Results and Analysis}

\subsection{NGC 1433}

Figure 1 shows contours of the $\mathrm{H}$ I column density overlaid on a $B$-band CTIO $1.5 \mathrm{~m}$ CCD image of NGC 1433, the latter displayed logarithmically to bring out the faint outer pseudoring as well as the inner ring, nuclear ring, and short "plume" features leading the bar. As Figure 1 shows, all these features are remarkably well matched to $\mathrm{H}$ I counterparts, while the bar itself, and the regions between the rings, are relatively devoid of neutral atomic hydrogen.

We have overlaid isovelocity contours from the velocity field of NGC 1433 on the H I column density map in Figure 2. The "spider" pattern characteristic of a disk in uniform circular motion is evident, but modulated by a lack of gas in the inter-ring regions with which to trace the kinematics, and kinks in high density regions indicative of non-circular motions there. There is a very steep velocity gradient within the central $1^{\prime}$ or so (most likely flattened somewhat by beam smoothing effects), while closed contours on the inner ring minor axis imply that the rotation curve turns over at this point. In order to determine a sensible rotation curve from this velocity field using the ROCUR algorithm (Begeman 1987) within AIPs, it was necessary to fix the disk inclination, as well 


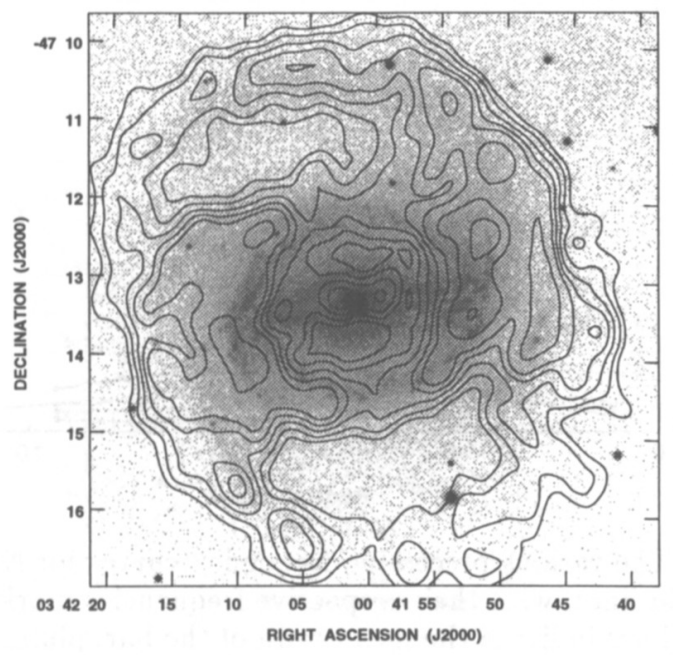

Figure 1. Contours of the $\mathrm{H}$ I column density overlaid on a $B$-band image of NGC 1433. The contour levels represent projected column densities of $1.4,1.9,2.5,3.2,3.8,5.0$, and $6.3 \times 10^{20} \mathrm{~cm}^{-2}$.

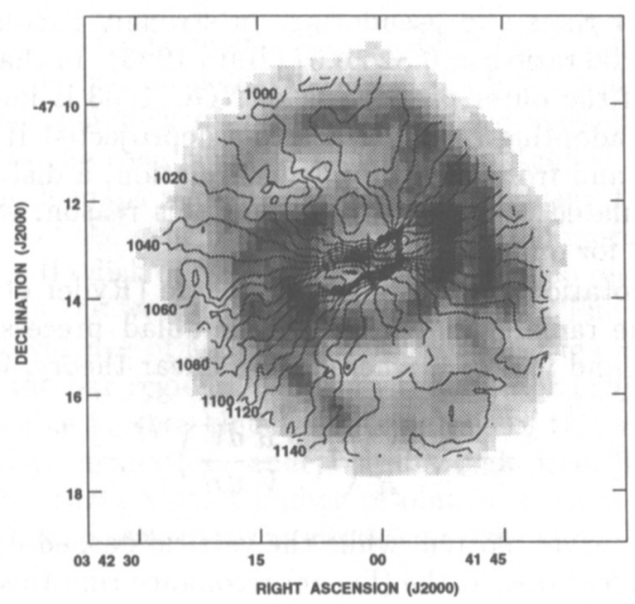

Figure 2. Isovelocity contours overlaid on the $\mathrm{H} \mathrm{I}$ column density map of NGC 1433. The contours are at intervals of $10 \mathrm{~km} \mathrm{~s}^{-1}$ and major velocity contours are marked. The cross marks the position of the dynamical center, as determined from the ROCUR analysis (see text). 


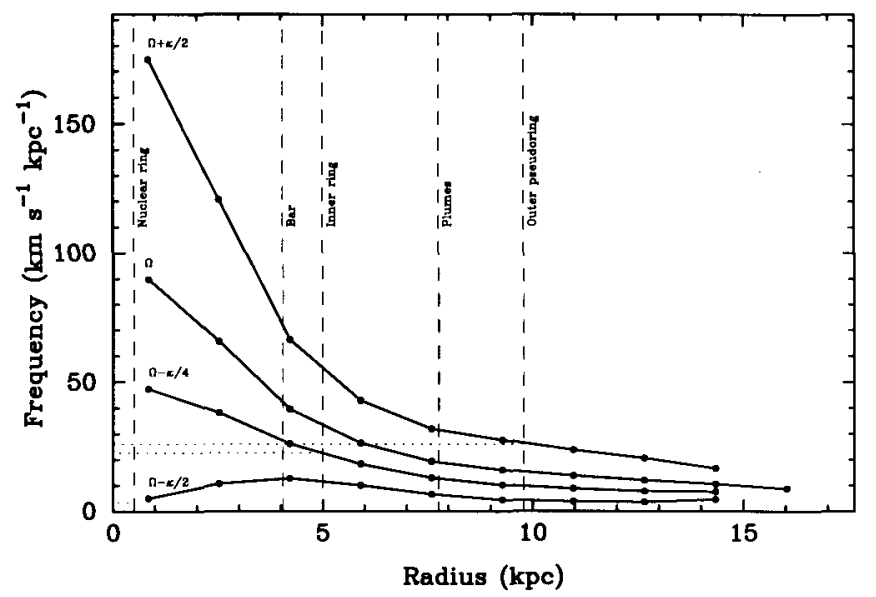

Figure 3. The various precession frequency curves for NGC 1433 are shown as solid lines, with their respective frequencies marked. The vertical dashed lines indicate the mean radii of the bar, plumes, and rings, and the horizontal dotted lines mark the bar pattern speed implied if the rings do correspond to specific resonances.

as the position of the dynamical center and systemic velocity, leaving both the rotational velocity and the line-of-nodes position angle free to be fitted.

The choice of a global disk inclination is complicated by the statistical evidence that outer rings and pseudorings in strongly barred galaxies have an average intrinsic axial ratio $q=0.82 \pm 0.07$ (Buta 1995). In that event, NGC 1433 has $i \sim 20^{\circ}$, but if the outer pseudoring of NGC 1433 is indeed circular, then $i=33^{\circ}$. However, adopting $i=20^{\circ}$ leads to a deprojected $\mathrm{H} \mathrm{I}$ velocity width of some $450 \mathrm{~km} \mathrm{~s}^{-1}$, and from the Tully-Fisher relation, a distance nearly double the 11.6 Mpc tabulated by Tully (1988). For this reason, we favor the higher inclination $i=33^{\circ}$ for our kinematical modeling.

The derived rotation curve is given elsewhere (Ryder et al. 1995), and we show here only the radial variation of the Lindblad precession frequencies $\Omega$, $\Omega+\kappa / 2, \Omega-\kappa / 2$, and $\Omega-\kappa / 4$, where in the linear theory, $\Omega=V / R$ and

$$
\kappa=\sqrt{2} \frac{V}{R}\left(1+\frac{R}{V} \frac{d V}{d R}\right)^{1 / 2}
$$

Figure 3 has these curves plotted, while the vertical dashed lines mark the mean radii of the optical features. If the (linear) resonance ring theory is correct, then each of the resonance curves ought to intersect the vertical line marking their associated ring at the same angular frequency value, namely the bar pattern speed $\Omega_{\mathrm{p}}$. Both the inner ring and the outer pseudoring are in fairly good agreement on $\Omega_{\mathrm{p}}$, while the $\Omega-\kappa / 2$ curve is dominated by beam smoothing. Bearing in mind the uncertainties in inclination, ring location relative to resonances, etc., we conservatively propose that $\Omega_{\mathrm{p}}=26 \pm 5 \mathrm{~km} \mathrm{~s}^{-1} \mathrm{kpc}^{-1}$ in NGC 1433 at the current epoch, placing corotation at $\sim 1.5 R_{\mathrm{bar}}$, just beyond the inner ring. 


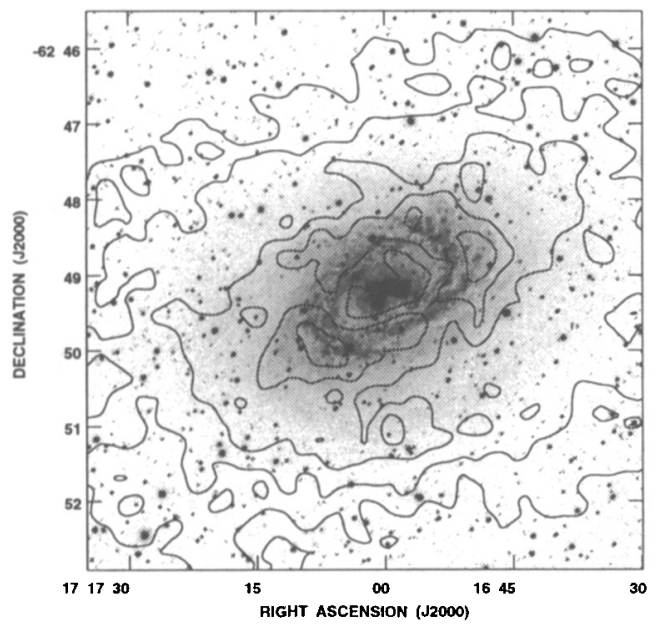

Figure 4. Contours of the H I column density overlaid on a $B$-band image of NGC 6300. The contour levels represent projected column densities of $0.6,2.0,4.9,7.9$, and $10.8 \times 10^{20} \mathrm{~cm}^{-2}$.

\subsection{NGC 6300}

Figure 4 shows contours of the H I column density in NGC 6300 superimposed on a logarithmic $B$-band CCD image. Again, the $\mathrm{H}$ I distribution has a prominent ring, which in this case is slightly larger in diameter and broader than the stellar ring, while the gas disk overall is much more extended relative to the optical disk than was the case in NGC 1433. This extended disk and its kinematics are best seen in maps made using natural weighting to enhance surface brightness sensitivity at the expense of some resolution (Figure 5). Aside from a $20^{\circ}$ warp in the outer H I disk of NGC 6300 , this figure also reveals a loose "tail" of gas emanating from the western edge of the gas disk. The uniform-weighted velocity field shows evidence of non-circular motions in the center, but these do not extend beyond the bar region. Owing to the greater projected gas column densities, it was possible to do a kinematical analysis of this velocity field keeping rotational velocity, inclination, and position angle free. By combining the extended $\mathrm{H}$ I rotation curve with a higher resolution optical one for the inner disk (Buta 1987), we can now plot the precession frequency loci in NGC 6300 (Figure 6).

If the inner ring is associated with the inner second harmonic resonance, then $\Omega_{\mathrm{p}}=27 \pm 8 \mathrm{~km} \mathrm{~s}^{-1} \mathrm{kpc}^{-1}$. However, there is no sign of the expected outer ring or nuclear ring for this $\Omega_{\mathrm{p}}$. Rather than finding fault with the resonance-ring theory, we propose instead that NGC 6300 is still in the course of evolving from a barred spiral to a mature resonance-ring galaxy, with NGC 1433 representing the pinnacle of this evolution. Support for this scenario comes from the optical bar-ring misalignment, the $\mathrm{H}$ I tail (evidence of ongoing mass accretion?), and a 


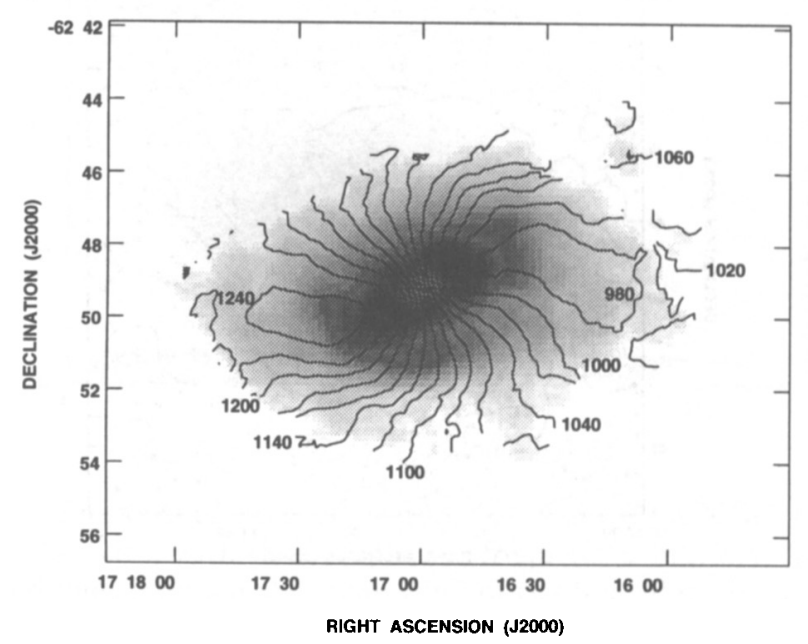

Figure 5. Isovelocity contours overlaid on the (natural-weighted) $\mathrm{H}$ I column density map of NGC 6300. The contours are at intervals of $20 \mathrm{~km} \mathrm{~s}^{-1}$ and major velocity contours are marked. The cross marks the position of the dynamical center.

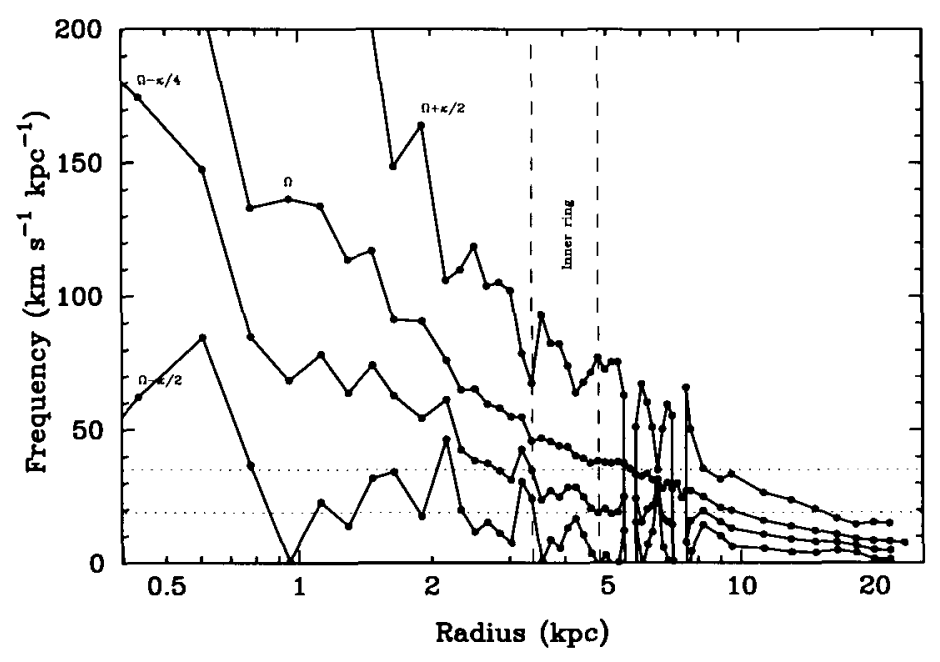

Figure 6. The various precession frequency curves for NGC 6300 plotted on a logarithmic radial scale. The vertical dashed lines indicate the approximate extent of the inner ring, and the horizontal dotted lines mark the possible bar pattern speed range implied if this ring was in fact associated with the inner second harmonic resonance. 
better match between the sites of recent massive star formation with the $\mathrm{H}$ I ring than with the old stellar pseudoring.

Acknowledgments. We thank the ATCA Time Allocation Committee for their support of this project. We wish to acknowledge support from the Alabama Space Grant Consortium, EPSCoR grant EHR-9108761, and DGAPA/UNAM grant IN107094.

\section{References}

Begeman, K. 1987, Ph.D. thesis, University of Groningen

Buta, R. 1986, ApJS, 61, 631

Buta, R. 1987, ApJS, 64, 383

Buta, R. 1995, ApJS, 96, 39

Contopoulos, G. \& Papayannopoulos, T. 1980, A\&A, 92, 33

Mulder, P. S. \& van Driel, W. 1993, A\&A, 272, 63

Ryder, S. D., Buta, R., Toledo, H., Shukla, H., Staveley-Smith, L., \& Walsh, W. 1995, ApJ, submitted

Schwarz, M. P. 1981, ApJ, 247, 77

Tully, R. B. 1988, Nearby Galaxies Catalogue, Cambridge: Cambridge University Press

van Driel, W., Rots, A. H., \& van Woerden, H. 1988, A\&A, 204, 39

van Driel, W. \& Buta, R. 1991, A\&A, 245, 7

\section{Discussion}

J. Sellwood: The linear theory is not appropriate in a strong-bar case, and in the presence of non-circular motions.

S. Ryder: True, but then our large beamsize makes it impossible to test the association between nuclear rings and $\Omega-\kappa / 2$ in the bar region anyway.

$J$. Knapen: Can you comment on the behavior of the molecular component of the neutral gas in these galaxies?

S. Ryder: I understand F. Combes has detected CO in NGC 1433 with SEST, but at $45^{\prime \prime}$ resolution, it will be difficult to tell if it lies in the bar, the rings, or elsewhere.

$W$. van Driel: The stubby tail of NGC 6300 is interesting, is it a sign of interaction? It reminds me a bit of the $\mathrm{H}$ I in the isolated, flocculent Sa NGC 3098, though the velocity field there is completely regular.

S. Ryder: A search with NED turned up no candidates for an interacting object within $30^{\prime}$ of NGC 6300 . 